\title{
A Survey and Comparison of SDN Based Traffic Management Techniques
}

\author{
Nandhini R. ${ }^{1} \&$ Evangelin Sonia S.V. ${ }^{2}$ \\ ${ }^{I}$ PG Student, ${ }^{2}$ Associate Professor, Department of Computer Science \& Engineering, Sri Shakthi Institute of Engineering and \\ Technology (Autonomous), Coimbatore, Tamil Nadu, India.
}

\section{ABSTRACT}

A distributed system can be viewed as an environment in which, number of computers/nodes are connected and resources are shared among these computers/nodes. But unfortunately, distributed systems often face the problem of traffic, which can degrade the performance of the system. Traffic management is used to improve scalability and overall system throughput in distributed systems using Software Defined Network (SDN) based systems. Traffic management improves system performance by dividing the work traffic effectively among the participating computers/nodes. Many algorithms were proposed for traffic management and their performance is measured based on certain parameters such as response time, resource utilization, and fault tolerance. Traffic management algorithms are broadly classified into two categories- scheduling and machine learning traffic management. This work presents the study of performance analysis of traffic management algorithms. This analysis can further help in the design of new algorithms. However, when multiple servers are assigned to compile the mysterious code, different kinds of techniques are used. One common example is traffic management. The processes are managed based on power efficiency, networking bandwidth, Processor speed. The desired outpu will again send back to the developer. If multiple programs have to be compiled then appropriate technique such as scheduling algorithm is used. So the compilation process becomes faster and also the other process can get a chance to compile. SDN based clustering algorithm based on Simulated Annealing whose main goal is to increase network lifetime while maintaining adequate sensing coverage in scenarios where sensor nodes produce uniform or non-uniform data traffic.

Keywords: Traffic Management, Scheduling, Machine learning, Clustering, SDN.

\section{Introduction}

The capacity to keep up administration without effect during any number of concurrent disappointments is named as high accessibility. It is broadly engaged with all parts of life, as it fills numerous needs like correspondence, business, diversion, training, informal community and so on. With this exceptional advancement in PC innovation, the interest of fast handling has raised thus the need for high versatility, accessibility, and quick reaction. The compiler will aggregate the code as needs be program precision, intricacy, and other related things and elegance imprints will be given to the specific engineer [5]. At the server-side, we need to plan all these programs to get wanted yield to the customer side in less time.

The fundamental topic behind this coder or designer composes the code as indicated by a given prerequisite and submits. It answers to customer demand without the customer regularly thinking about the inside conveyance. At the point when the quantity of servers develops, the likelihood of a disappointment is expanding and such disappointments must be dealt with cautiously. Numerous clients are presenting their programs at once. A traffic-management calculation ought to be general and straightforward to the applications, additionally; it ought to give the least overhead to the framework. The undertaking line which will simply line your answer and specialist hub will get an assignment and run it and update the outcomes. Aside from sharing information and I/O gadgets, appropriated frameworks additionally share the computational power among hubs, which improves the framework's presentation [2].

To expand the effectiveness of the system various servers are sent associated with one another to frame a server ranch. Today a huge number of clients use the web; this quick development has to lead to the capacity of huge measure of information and ascends in web traffic. Traffic management expands accessibility, improves execution 
by expanding unwavering quality, builds throughput, looks after, steadiness, enhances asset use and gives deficiency tolerant ability. The traffic management is characterized as a procedure of dispensing the complete outstanding task at hand to the individual hubs of the circulated frameworks to improve asset use and reaction time, likewise staying away from the condition in which a few hubs are over-burden while others are under stacked or server disappointment. Hackerrank is one top coding site that is utilized by numerous engineers.

Traffic management likewise gives level scaling e.g., adding figuring assets to address expanded traffics. The traffic management is exceptionally basic in dispersed processing frameworks to improve the nature of administration by managing changes in the traffic which happen after some time which prompts upgrade the exhibition of frameworks. These sites are most prevalent because a group of individuals is picking up consideration towards these including designer, IT organizations, additionally developer specialist. Innovation has changed the life standard and web administrations have a vital influence on this innovation.

The approaching solicitations are ideally disseminated among accessible framework assets to maintain a strategic distance from asset bottlenecks just as to all the more likely use accessible assets. These components expanded the pattern of utilizing appropriated frameworks. The way toward dispersing customer demand among servers is performed by the traffic management procedure. Customer solicitation is moved to one of the back end servers in the server ranch. The web is one such administration that has associated with everyone in a manner that was scarcely possible.

As a rule, as the intricacy of appropriated frameworks develops, their traffic management requires greater progression usefulness, for example, the capacity to endure shortcomings, put in new traffic management calculations at run time and to make reproduction to deal with curvy customers. Dispersed frameworks execution and adaptability will be influenced because of the absence of the above functionalities [1].

The traffic management procedure is characterized in three standards, the initial one is area rule which gives data about the asset space to be incorporated into the adjusting activities and the second principle is conveyance rule which is utilized if redistribution among hubs is required. An appropriated framework comprises of figuring hubs associated together by a correspondence organize. This has further lead to the issues of system blockage, web administration delay and moderate reaction time. A planning calculation utilized for getting a reasonable time for the specific program when there is a high burden and the client will get the yield in less time. Numerous methods can be executed in these sites. The clients get a solitary framework picture of this physically dispersed framework. The Coding site fundamentally centers on programming difficulties where the designer satisfies the prerequisite according to detail.

\section{Related Work}

The creators improved the server execution in their work. Atul et al introduced the correlation of different transformative quest techniques created for inquiry improvement on web servers [3]. Results demonstrate that the exhibition of PSO is better when contrasted with different calculations but it stays behind in handling time. Developmental calculations are broke down and analyzed based on their conduct, adaptability and transmission 
techniques and so forth. The technique indicated a high pace of adaptability however there is have to lead much research around there. In their work, the creator proposed a functioning subterranean insect at the customer side and the server-side both. The customer insect is in charge of the solicitation through the server subterranean insect is in charge of answering the solicitation.

Fengyun et al introduced a straightforward and compelling methodology of traffic management for MMORPGs and web-based games that utilize bunches of server for high versatility [1]. Sandeep et al exhibited the presentation investigation of scheduling and machine learning traffic management calculations [2]. Dimple and Atul proposed a subterranean insect-based structure to adjust the traffic [4]. The social way to deal with traffic management is mulled over when contrasted with topographical methodology as it experiences the issue of swarming.

Correlation is done the different parameters of over-burden dismissal, adaptation to internal failure, precision and soundness and so forth. Determination of traffic management calculation depends on the circumstance in which outstanding traffic is relegated for example at run time or scheduling time. Scheduling calculations are demonstrated to be progressively steady as a contrast with machine learning traffic management calculations.

\section{Problem Definition}

Traffic management is one of the most significant issues in accomplishing elite in appropriated frameworks which may comprise numerous heterogeneous assets associated with at least one system. At the point when the interest of figuring force builds, the traffic management issue winds up significant. A significant issue in the dispersed framework condition is the exhibition in which the reaction time of a solicitation gets limited. The problem here is to choose how to accomplish parity in the traffic dissemination between processors with the goal that the calculation is finished in the most limited conceivable time. This circumstance can prompt a poor framework execution. The issue of undertaking and traffic management in the disseminated framework is the most significant and testing zones of research. In these dispersed frameworks condition, it is workable for certain PCs to be vigorously stacked while others are daintily stacked.

\section{Overview of Traffic Management}

Traffic management performs the following functions and it is given in figure 1.

(a) Intercepts the traffic sent by the client.

(b) Divides the heap among individual hubs and chooses which hub ought to get and process the solicitation.

(c) Maintains the log of accessible hubs and guarantees that they are reacting to the traffic. If not they are removed from the pivot.

(d) Provide repetition by sending separate units for disappointments.

Traffic management calculation endeavor to adjust the traffic on the entire framework by relocating the remaining task at hand from intensely stacked hubs to softly stacked hubs to upgrade the framework exhibitions, traffic management calculations can be arranged into 2 classifications as scheduling and machine learning traffic management algorithms. Most significant errands in traffic management are to decide traffic on every hub and 
afterward taking the choice to move to process starting with one hub then onto the next. The traffic balancer is founded on two qualities. To begin with, dole out traffic to the best competitor hub, and second, relocation of traffic from intensely stacked hub to gently stacked hub [5].

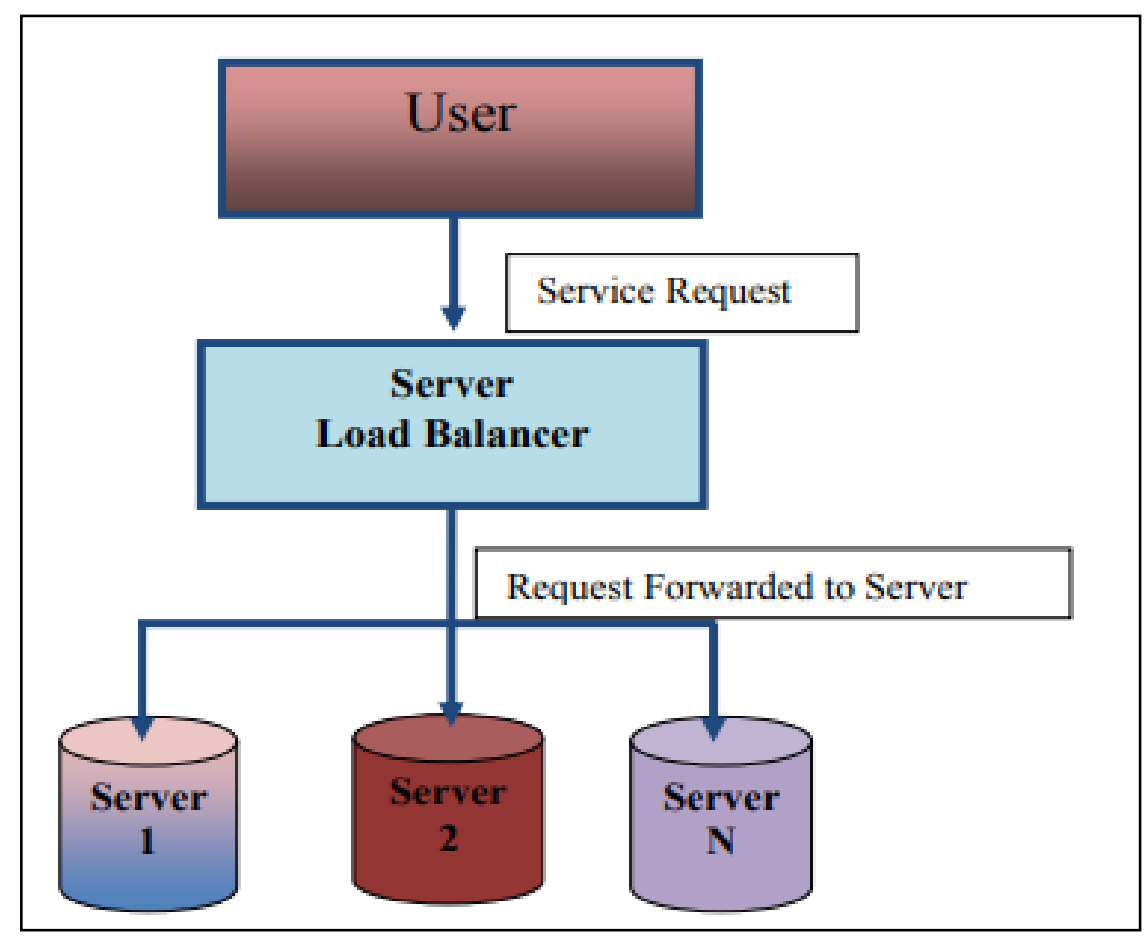

Figure No: 1 Traffic Balancer

\subsection{Scheduling Approach in Traffic Management}

Scheduling traffic management calculations to disperse the burden dependent on a fixed arrangement of guidelines. The presentation of the processor is resolved during the arrangement time. The primary favorable circumstances of the scheduling traffic management calculations are that they limit the execution time of the procedures and they are an easier plan and simple usage. It doesn't consider the genuine current framework status [6]. These standards are identified with burden type, such as handling power prerequisites, memory necessity, and so on. Henceforth, they can't guarantee whether equalization is even or not. Likewise, the significant impediment of them is; they don't check the traffic on different hubs. So they have lower execution. The objective of scheduling the traffic management technique is to diminish the execution time, limiting the correspondence delays.

(1) In the round-robin, all occupations are appointed to a rundown of slave processors accessible dependent on turning requests, implying that picking a processor is performed in an arrangement and will have returned to the principal processor if the last processor has been reached. It is reasonable for the webserver application where HTTP solicitations are comparable. The fundamental favorable position of Round Robin calculation is that it doesn't cause overhead in the framework since it needn't bother with entombing process correspondence. In Round Robin calculation, the ace hub conveys employments equitably to all slave processors. Then again it does not require some investment in thought so it does not accomplish great execution. 
(2) The standard that is pursued to pick the slave processor is to dole out the activity to the processor that has the least burden. The Central Manager Algorithm utilizes a focal hub as a facilitator to appropriate the outstanding task at hand among the slave processors. The focal processor can assemble all slave processors traffic data and take the choice of traffic management relying upon this data so we expected a decent presentation when applying this calculation. The principle impediment of this sort is the high level of between procedure correspondences that could make a bottleneck state.

(3) Randomized calculation choosiest the slave processors utilizing arbitrary numbers. The randomized calculation can acquire the best execution among all traffic management calculations for specific particular reason applications. The slave processors are picked arbitrarily following arbitrary numbers produced relying upon a measurement circulation.

(4) In Threshold calculation, the procedures are allocated quickly to the server hubs upon creation. Every server hub keeps a duplicate of the framework's present traffic. Server hubs for a new position are chosen locally without sending remote messages. The traffic of a processor can be described by one of the three levels: under stacked, medium, and over-burden.

\subsection{Machine Learning-Based SDN Approach in Traffic Management}

The motivation of the work pronounced in this work is the development of SDN network management software for corporation networks that uses machine gaining knowledge of. Another difference is that statistics are obtained directly in the controller, wherein the control aircraft resides, establishing the opportunity to directly use learned records, just like the class of site visitors, for network management. Machine Learning (ML) strategies can also be used for traffic classification and several works were proposed. SDN presents new methods to perform those operations. The first step in the direction of this aim is to examine how traffic records can be found out by using an SDN controller the usage of SDN mechanisms [7]. Open Flow has built-in flow information and the forwarding guidelines (Flow Entries) installed within the switches suit packets into flows at numerous feasible granularities.

There is also a variety of flexibility in how statistics can be accrued. In supervised to know there's a need to gain labeled education records-sets, something that can be difficult in computer networks because of the difficulty in acquiring as it should be annotated community waft samples throughout a huge variety of applications and the charge at which new programs can appear. Traffic category may be finished using supervised to know with algorithms which include Support Vector Machines (SVM), neural networks and decision tree.

It can be customized by way of defining specific kinds of flow granularities for exclusive sorts of visitors and a customizable variety of packets may be analyzed in the controller before putting in a glide rule within the switch to gather its information. There is also more than one level at which such information can be accumulated, from getting admission to switches where the quantity of flows is smaller to core switches or gateway switches in which an excessive number of traffic flows can exist. If the facts on the site visitors are learned on-line, as an instance the category of visitors glide after a quick initial range of packets, the controller can right now act with the flow-through applying new forwarding guidelines to its packets [6]. 
Machine learning traffic management should be possible in two unique ways: conveyed and non-disseminated. A focal hub in the bunch is picked by utilizing race strategy. In an agreeable structure, all hubs have a shared objective. The Machine learning Traffic Management is more mind-boggling than scheduling however it has apriority as opposed to scheduling traffic management and it gives preferable exhibition over the scheduling one. The choice of adjusting the traffic is taken dependent on the present status of the framework.

\subsection{Clustering Approach in Traffic Management}

Progressive plans can be utilized for a homogeneous system or the heterogeneous system. Some calculation which works in the guideline of various leveled plans is LEACH, HEED, and EECS. The determination of groups and relegating a few errands to cluster heads can influence the lifetime, versatility, and productivity. Subsequently, to frame a group between various hubs and to pick cluster heads, there are diverse grouping plans accessible points. In the ongoing years, researchers have discovered bunching of hubs will lessen the power utilization [8]. Typically Heterogeneous systems are two-level systems, where Cluster head at the main level and Sensor hubs are at the following level.

(a) In this manner, the LEACH convention improves vitality productivity and broadens the lifetime of the sensor node. This convention utilizes the randomization cluster head choice strategy relying upon the vitality accessible by the sensor hubs. During the cluster head determination, every hub will create an arbitrary number somewhere in the range of 0 and 1. LEACH (Low Energy Adaptive Clustering Hierarchy) is a group-based convention, which is utilized in WSN. The irregular number produced by the sensor hub will be contrasted and the edge work. It utilizes the clustering idea to circulate the vitality devouring of the hub by the irregular pivot of group heads. If the worth is not exactly the edge esteem, at that point the specific hub will be chosen as the Cluster Head. Thusly, the vitality spent by group heads could be diminished to draw out system lifetime.

(b) HEED (Hybrid Energy-Efficient Distributed) is a Clustering calculation that picks the bunch head with the accompanying parameters like remaining vitality and intra-group correspondence cost. To choose a cluster head, every hub will figure the lingering vitality. It permits a single jump and multihop correspondence. Every sensor hub will speak with single bounce and multihop correspondence between-group head and base station. To frame a group, intra-cluster correspondence cost is determined.

(c) As a rule, the group that is in the long-range will require more vitality when contrasted with the cluster which is close to the base station. This convention beat the above issue by disseminating the vitality over the system. At first, every hub communicates its remaining vitality to its neighboring hub. This outcome is better asset use and the system lifetime will be broadened. Similarly, the development of the group fluctuates with different conventions. At the point when the sensor hub gets this message it contrasts and its lingering vitality and it is more than the other sensor hub, at that point the specific sensor hub will be chosen as the cluster head. EECS (Energy Efficient Clustering Scheme) Algorithm is a grouping calculation that alternately chooses the cluster head when contrasted and the LEACH convention. This convention bolsters machine learning measuring the group and not on the least separation of hubs. The comparisons of various traffic management algorithms are illustrated in table 1. 
Asian Journal of Applied Science and Technology

Volume 4, Issue 3, Pages 09-16, July-September 2020

Table No: 1 Comparison of Traffic Management Techniques

\begin{tabular}{|c|c|c|c|c|c|}
\hline S. No & Methods & Overhead & Throughput & $\begin{array}{c}\text { Response } \\
\text { Time }\end{array}$ & $\begin{array}{c}\text { Fault } \\
\text { Tolerance }\end{array}$ \\
\hline $\mathbf{1}$ & Scheduling & High & Low & Low & Moderate \\
\hline $\mathbf{2}$ & Machine learning & Low & Moderate & High & High \\
\hline $\mathbf{3}$ & Clustering & Low & High & High & High \\
\hline
\end{tabular}

\section{Conclusion}

A proficient clustering calculation ought to be picked for the cluster arrangement and choice of cluster head. The traffic balance calculation ought to be utilized in the system to disseminate the traffic among the neighbored hubs. In this work, the examination of various traffic management calculations is carried based on specific parameters. The traffic management is required to disseminate the remaining traffic uniformly over all hubs to accomplish elite; with least overheads. This examination further can likewise help in structuring new traffic management calculations. The above examination demonstrates that scheduling traffic management calculations are steadier than machine learning calculations however because of the capacity of performing exactly in disseminated frameworks; the machine learning traffic management is picked over scheduling traffic management calculations. By utilizing the effective grouping procedure and traffic management calculation, control devouring in the system can be limited and the lifetime of the system can be expanded. With appropriate traffic management holding up time can be kept to a base which will further expand the reaction time.

\section{References}

[1] Fengun, Simon and Graham," Traffic management for massively multiplayer online games ", Netgames 06, Oct 30-31, 2006, Singapore.

[2] Sandeep Sharma, S.Singh and Meenakshi, "Performance analysis of traffic management algorithms", World Academy of Science, 2008.

[3] Atul Garg and Dimple Juneja, "A Comparison and analysis of various extended techniques of query optimization", IJICT Vol-3 no-3 July-2012.

[4] Dimple Juneja and Atul Garg," Collective Intelligence based framework for traffic management of web servers", IJICT, Vol. 3 No-1 Jan-2012.

[5] Soundarabai.P, Sahai.R, Venugopal.R, Patnaik.L., "Comparative Study On Traffic Management Techniques In Distributed Systems", International Journal of Information Technology and Knowledge Management, Volume 6, No. 1, pp. 53-60, December 2012. 
[6] Beniwal.R, Garg.A, "A comparative study of scheduling and machine learning Traffic Management Algorithms", International Journal of Advance Research in Computer Science and Management Studies Volume 2, Issue 12 ISSN: 2327782, December 2014.

[7] T. T. Nguyen and G. Armitage, "A survey of techniques for internet traffic classification using machine learning”, IEEE Communications Surveys \& Tutorials, 10(4):56-76, 2008.

[8] Rajani.SH, Garg.N, "A Clustered Approach for Traffic Management in Distributed Systems, SSRG International Journal of Mobile Computing \& Application, Volume 2 Issue 1, Jan to Feb 2015. 